\title{
Overview of the HiLASE project: high average power pulsed DPSSL systems for research and industry
}

\author{
M. Divoky ${ }^{1}$, M. Smrz ${ }^{1}$, M. Chyla ${ }^{1}$, P. Sikocinski ${ }^{1}$, P. Severova ${ }^{1}$, O. Novak ${ }^{1}$, J. Huynh ${ }^{1}$, S.S. Nagisetty ${ }^{1}$, \\ T. Miura ${ }^{1}$, J. Pilař ${ }^{1}$, O. Slezak ${ }^{1}$, M. Sawicka ${ }^{1}$, V. Jambunathan ${ }^{1}$, J. Vanda ${ }^{1}$, A. Endo ${ }^{1}$, A. Lucianetti ${ }^{1}$, \\ D. Rostohar ${ }^{1}$, P.D. Mason ${ }^{2}$, P.J. Phillips ${ }^{2}, K_{\text {Krtel }}^{2}$, S. Banerjee ${ }^{2}$, C. Hernandez-Gomez ${ }^{2}$, J.L. Collier ${ }^{2}$, and \\ T. Mocek ${ }^{1}$ \\ ${ }^{1}$ HiLASE, Institute of Physics, AS CR, v.v.i., Na Slovance 2, 18221 Prague, Czech Republic \\ ${ }^{2}$ STFC Rutherford Appleton Laboratory, Didcot OX11 OQX, United Kingdom \\ (Received 28 February 2014; revised 21 April 2014; accepted 24 April 2014)
}

\begin{abstract}
An overview of the Czech national R\&D project HiLASE (High average power pulsed laser) is presented. The project focuses on the development of advanced high repetition rate, diode pumped solid state laser (DPSSL) systems with energies in the range from $\mathrm{mJ}$ to $100 \mathrm{~J}$ and repetition rates in the range from $10 \mathrm{~Hz}$ to $100 \mathrm{kHz}$. Some applications of these lasers in research and hi-tech industry are also presented.
\end{abstract}

Keywords: DPSSL; $\mathrm{Yb}^{3+}$ :YAG; thin-disk; multi-slab; pulsed high average power laser

\section{Introduction}

Efficient diode pumping of solid-state lasers (DPSSL) has enabled lasers to reach $\mathrm{CW}$ output powers in the region of $100 \mathrm{~kW}^{[1,2]}$. High energy pulsed DPSSLs are far behind the $\mathrm{CW}$ systems in respect of average power, but the development of new technology [thin disk, multi-slab] will enable operation at comparable average powers. Many laser projects are trying to reach the barrier of $1 \mathrm{~kW}$ average power in pulsed operation using either thin disks at high repetition rates ${ }^{[3-7]}$ or a high energy and lower repetition rate with conventional designs ${ }^{[8]}$, TRAM (Total Reflection Active Mirror $)^{[9]}$, thick disks ${ }^{[10]}$ or multi-slabs ${ }^{[11,12]}$. The HiLASE (High average power pulsed laser) project is aimed at the development of the next generation of pulsed DPSSL for hi-tech industrial applications, such as laser-induced damage testing, extreme ultra-violet (EUV) light generation, surface cleaning, precise manufacturing, laser peening, etc. HiLASE will be a user facility with several laser systems, with output parameters ranging from a few picosecond pulses with energies of $5 \mathrm{~mJ}-0.5 \mathrm{~J}$ and repetition rates of $1-100 \mathrm{kHz}$ (thin disk technology) to systems with $100 \mathrm{~J}$ output energy in nanosecond pulses with a repetition rate of $10 \mathrm{~Hz}$ (multi-slab technology).

In this paper, an overview of the HiLASE activities, including laser development and laser applications, will be presented.

Correspondence to: Email: divoky@fzu.cz

\section{Kilowatt-class thin disk laser system}

For efficient generation of EUV and mid-IR light, a laser producing several $\mathrm{mJ}$ per pulse at a repetition rate of $1-100 \mathrm{kHz}$ is required. For industrial applications, it is important to realize a robust, compact, and low-cost alternative to Ti:sapphire-based pulsed laser systems. Thindisk lasers with their feature of a high pulse energy in the sub-picosecond region are one of the best devices suited for this application.

A thin-disk laser is based on an amplifier concept ${ }^{[13]}$ that utilizes a very thin $(\sim 200 \mu \mathrm{m})$ laser active medium on a heatsink. The front face of the disk is AR coated, while the back face is HR coated and the disk works as an active mirror. Since the diameter of the disk is much larger than its thickness, the heat flux is mostly axial and the transverse temperature gradient is low. The path of the beam in the disk is short and thermal lens effects and mechanical deformation do not affect the beam quality much. Additionally, a low material path minimizes nonlinear phenomena such as selffocusing.

On the other hand, the thinness of the disk causes minimal pump light absorption and laser light gain. Therefore, the number of passes of both pump and laser light must be high. The pump light is sequentially reflected back to the laser disk by a parabolic reflector and roof prisms (Figure 1), so the absorption can exceed $90 \%$. The number of laser light passes is usually increased in a regenerative cavity or in a multi-pass amplifier with many extraction passes. 


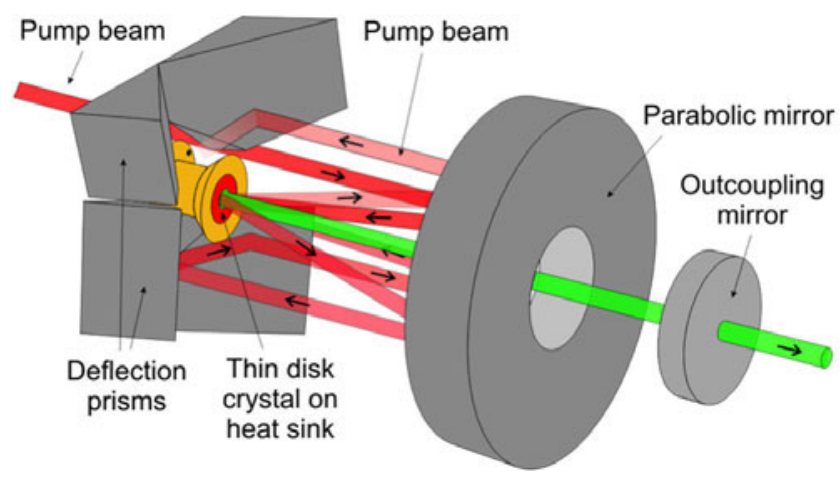

Figure 1. Schematic of the thin-disk cavity consisting of a parabolic mirror focusing the pump beam onto the thin-disk crystal. Multiple passes of the pump beam are made by means of deflection prisms. The cavity for laser beam extraction is formed by the thin-disk crystal and the outcoupling mirror [14]

Within the HiLASE project, three thin-disk-based $\mathrm{kW}$ class laser beamlines are being developed, each delivering different output parameters. Beamline A will deliver a $750 \mathrm{~mJ}$ pulse energy at a $1.75 \mathrm{kHz}$ repetition rate. This beamline is subcontracted to Dausinger and Giesen $\mathrm{GmbH}$ in order to reduce the overall project risk associated with the high demands. The HiLASE research group is developing Beamlines B and C with output parameters of $500 \mathrm{~mJ}$ at a $1 \mathrm{kHz}$ repetition rate and $5 \mathrm{~mJ}$ at a $100 \mathrm{kHz}$ repetition rate, respectively. All beamlines will provide a pulse duration of 1-3 ps. The output of Beamline B could be diverted into a $10 \mathrm{~Hz}$ repetition rate cryogenic amplifier that would later be upgraded to multi-joule output at repetition rates up to $120 \mathrm{~Hz}$. Figure 2 shows the block diagrams of each beamline in the HiLASE project.

\subsection{Beamline A}

Beamline A consists of a fiber front-end that includes a pulse stretcher, pulse picker, and optical isolator. The front-end produces laser pulses with an energy of $1 \mu \mathrm{J}$ at a repetition rate of $1.75 \mathrm{kHz}$. These pulses are further amplified in a regenerative amplifier to an energy around $150 \mathrm{~mJ}$, then in a linear amplifier to an energy around $0.9 \mathrm{~J}$. The amplified pulses are compressed in a grating pulse compressor to below 3 ps.

\subsection{Beamline B}

Beamline $\mathrm{B}^{[15]}$ starts with an Yb-doped fiber oscillator operating at a $50 \mathrm{MHz}$ repetition rate, delivering an output power of $2 \mathrm{~W}$ at a center wavelength of $1030 \mathrm{~nm}$ with a bandwidth of more than $20 \mathrm{~nm}$. Pulses from the fiber laser are stretched by a Martinez-type stretcher up to 500 ps. A gold-coated grating with a groove density of $17401 \mathrm{~mm}^{-1}$ was employed, providing a group delay dispersion of $1.41 \times$ $10^{8} \mathrm{fs}^{2} / \mathrm{rad}$. After the pulse stretching, pulses are coupled into the regenerative amplifier cavity, which contains an $\mathrm{Yb}^{3+}$ :YAG thin disk, a thin film polarizer (TFP), a Pockels cell with a $10 \mathrm{~mm} \times 10 \mathrm{~mm} \times 22 \mathrm{~mm}$ BBO crystal, highreflection mirrors, and a quarter-wave plate. Pulses from the stretcher are captured in the amplifier cavity when a quarterwave voltage of more than $10 \mathrm{kV}$ is applied to the Pockels cell, and they travel inside the cavity as long as the high voltage is applied. After amplification, the amplified pulse is ejected through the TFP and passes through an optical isolator consisting of a half-wave plate and a Faraday rotator. Afterwards, the amplified beam is diverted from the input beam by the polarizing beam splitter (PBS) and sent to the pulse compressor. A schematic of the current status of Beamline B is shown in Figure 3.

In order to reduce thermally induced stress between the heatsink and the gain media, $\mathrm{CuW}$ is adopted as the heatsink material, because it has similar linear expansion coefficient to YAG. The $\mathrm{Yb}^{3+}$ :YAG crystal, doped to 7 at.\%, has thickness of $0.22 \mathrm{~mm}$ and is soldered on the $\mathrm{CuW}$ heatsink using gold-tin solder. The mounted disk is pumped by a fiber-coupled diode laser module delivering an optical power

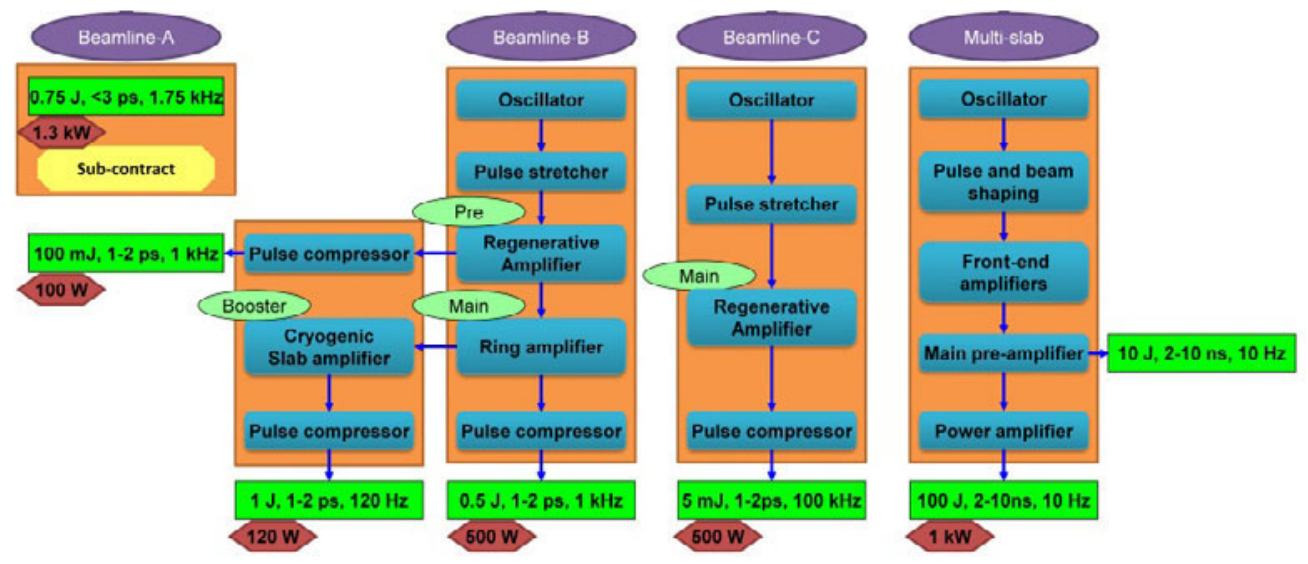

Figure 2. Overview of the HiLASE beamlines. 


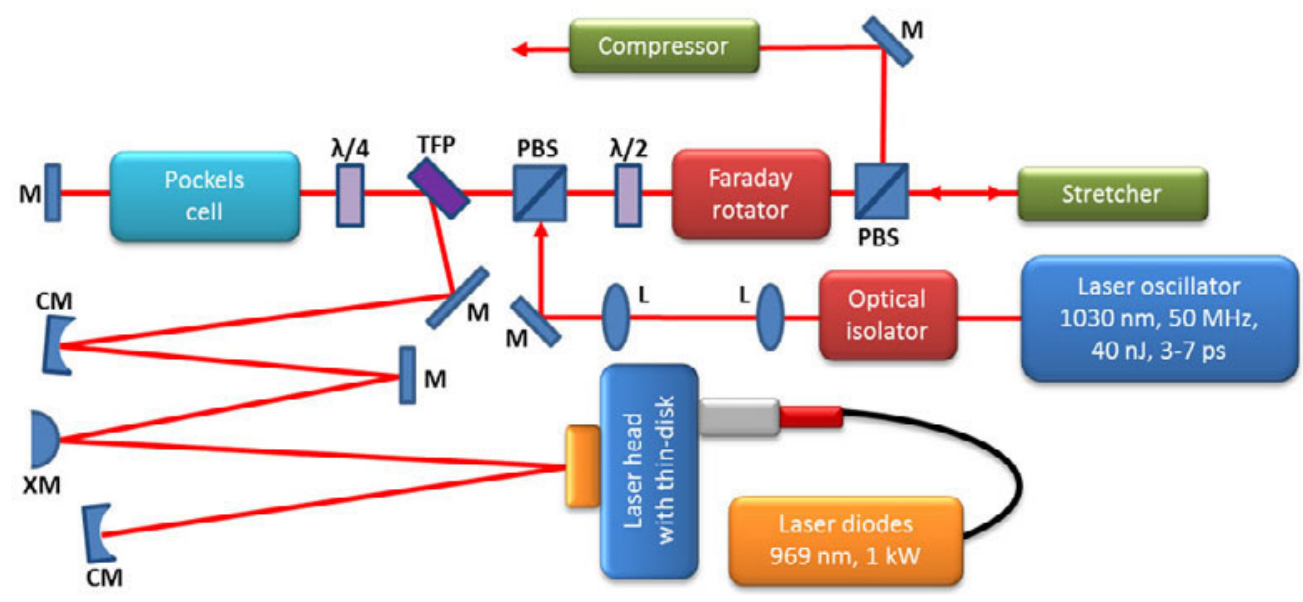

Figure 3. Schematic of the current status of Beamline B. Shown are flat mirrors (M), convex mirrors (XM), concave mirrors (CM), lenses (L), half and quarter waveplates $(\lambda / 2, \lambda / 4)$, PBS, and a thin film polarization beam splitter (DP).

of up to $1 \mathrm{~kW}$ at a wavelength of $969 \mathrm{~nm}$. The pump spot size on the disk was set to $4.8 \mathrm{~mm}$ to achieve an output of $45 \mathrm{~mJ}$, and the amplifier cavity was designed so that the cavity mode was matched to the designed pump spot size. The optical-to-optical efficiency was close to $20 \%$. The amplified laser pulses had a bandwidth of $1.5 \mathrm{~nm}$, so they could be compressed down to $1 \mathrm{ps}$.

The laser cavity will later be upgraded with a second thindisk head to reach an output energy of $100 \mathrm{~mJ}$. Additionally, the Martinez-type stretcher will be replaced with a fiberchirped Bragg grating stretcher that allows better control of dispersion and is more stable and compact. Finally, the amplified pulses will be directed to a second regenerative amplifier that will be constructed in 2014.

\subsection{Beamline $C$}

Beamline $\mathrm{C}^{[16]}$ is aimed at achieving a pulse energy of $5 \mathrm{~mJ}$ at a $100 \mathrm{kHz}$ repetition rate. In order to meet these requirements, an intense study has been conducted to develop a high repetition rate regenerative amplifier. The target specifications of Beamline $\mathrm{C}$ will be achieved after completing three milestones determined by the pulse energies, namely $0.5,2$, and $5 \mathrm{~mJ}$. The experimental setup for reaching the first milestone $(0.5 \mathrm{~mJ})$ is shown in Figure 4. The regenerative amplifier is seeded by an $\mathrm{Yb}^{3+}$-doped fiber oscillator, as in Beamline $\mathrm{B}$, but the pulses are stretched by a chirped volume Bragg grating (CVBG) up to $\sim 160$ ps. The dispersion of the CVBG is $60 \mathrm{ps} \mathrm{nm}^{-1}$. The FWHM spectral bandwidth and the clear aperture are $2.2 \pm 0.5 \mathrm{~nm}$ and $8 \mathrm{~mm} \times 8 \mathrm{~mm}$, respectively. The dimensions of the BBO crystal for the Pockels cell are $5 \mathrm{~mm} \times 5 \mathrm{~mm} \times 25 \mathrm{~mm}$ and its quarter-wave voltage is $5.2 \mathrm{kV}$. An $\mathrm{Yb}^{3+}$ :YAG thin-disk with a free aperture of $8 \mathrm{~mm}$ and a thickness of $220 \mu \mathrm{m}$ is installed in the laser head. The disk is pumped by a $1 \mathrm{~kW}$ fiber-coupled diode laser module at a wavelength of $969 \mathrm{~nm}$

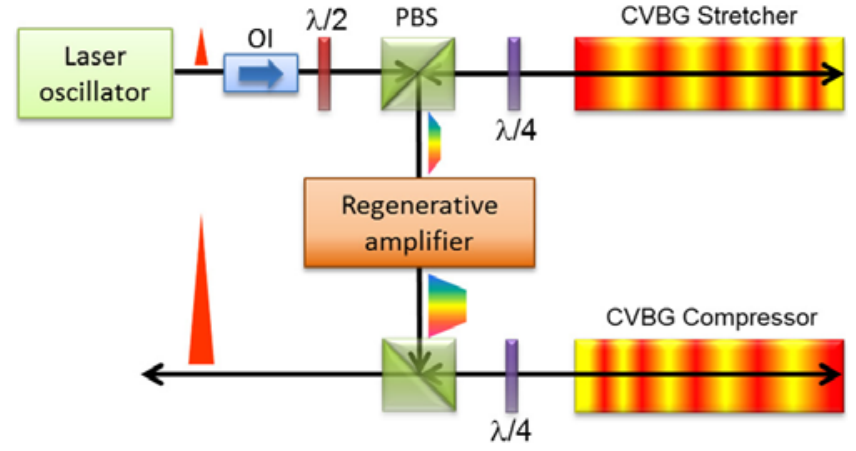

Figure 4. Schematic of the current status of Beamline C. Shown are an optical isolator (OI), $\lambda / 2, \lambda / 4$, PBS, and a CVBG stretcher and compressor.

with a pump spot diameter of $2.8 \mathrm{~mm}$. A schematic of the beamline is shown in Figure 4.

The observed output energy was $830 \mu \mathrm{J}$ at a $100 \mathrm{kHz}$ repetition rate. The low pulse energy enabled compression in a highly efficient CVBG compressor. The compressed pulse energy and the efficiency of the CVBG were $730 \mu \mathrm{J}$ and $88 \%$, respectively. The output pulse had a spectral bandwidth of $1.2 \mathrm{~nm}$ and was compressed only to $4 \mathrm{ps}$ pulse duration because the CVBG did not account for the dispersion of material in the path of the beam. By adding an additional diffraction grating compressor, the duration of the compressed pulses was decreased below 2 ps. The output energy will be increased by using more intense pump light and by modifying the thin-disk head and the cavity. Technical difficulties connected to further development of all the mentioned thin-disk beamlines are connected mostly to thermal management of the thin-disk modules and the availability of pump modules at a wavelength of $969 \mathrm{~nm}$.

The current status of thin-disk beamlines is indicated in Table 1. 
Table 1. Status of kW-class Thin-disk Beamlines.

\begin{tabular}{|c|c|c|c|c|}
\hline Laser system & Beamline A & Beamline B & Beamline C & Cryogenic beamline \\
\hline Completed & Front-end & $\begin{array}{l}\text { Regenerative amplifier with } \\
\text { one thin-disk head }\end{array}$ & $\begin{array}{l}\text { All, except high power pump } \\
\text { modules }\end{array}$ & None \\
\hline Under development & $\begin{array}{l}\text { Regenerative amplifier (May } \\
\text { 2014) }\end{array}$ & $\begin{array}{l}\text { Add second thin-disk head into } \\
\text { regenerative amplifier }\end{array}$ & Add high power pump modules & $10 \mathrm{~Hz}$ concept amplifier \\
\hline Achieved energy & $1 \mu \mathrm{J}$ & $45 \mathrm{~mJ}$ & $0.8 \mathrm{~mJ}$ & NA \\
\hline Next milestone energy & $150 \mathrm{~mJ}$ & $100 \mathrm{~mJ}$ & $2 \mathrm{~mJ}$ & $1 \mathrm{~J}(10 \mathrm{~Hz})$ \\
\hline Final energy & $750 \mathrm{~mJ}$ & $500 \mathrm{~mJ}$ & $5 \mathrm{~mJ}$ & $1 \mathrm{~J}(100 \mathrm{~Hz})$ \\
\hline Operational & Q2 2015 & Q2 2015 & Q2 2015 & 2016 \\
\hline
\end{tabular}

\section{Kilowatt-class multi-slab laser system}

To generate high energy pulses at low/moderate repetition rates, it is necessary to adopt an effective cooling mechanism and geometry. One of the solutions is to use an active medium in a slab geometry with active cooling of the slab faces, called a multi-slab, firstly adopted on the Mercury laser at Lawrence Livermore National Laboratory ${ }^{[17]}$. Room-temperature helium gas was used for cooling the slabs and $60 \mathrm{~J}$ at a $10 \mathrm{~Hz}$ repetition rate was obtained. The material of choice for next-generation high energy solid-state lasers is $\mathrm{Yb}^{3+}$ :YAG ceramic. However, $\mathrm{Yb}^{3+}: \mathrm{YAG}$ is a quasithree-level system that requires a high pump intensity for laser operation, thus increasing the number and cost of the pumping diodes. By cooling the crystal to low temperatures, the energy scheme changes to four levels, thus decreasing the threshold intensity by several orders of magnitude. Such a concept was introduced by DiPOLE ${ }^{[11]}$, where an energy of $10 \mathrm{~J}$ was obtained. The cooling of the slabs was done using helium gas at a temperature of around $150 \mathrm{~K}$. The next step is to demonstrate more than $100 \mathrm{~J}$ at a $10 \mathrm{~Hz}$ repetition rate, which will eventually lead to the delivery of 1 to $10 \mathrm{~kJ}$ pulses in a single beam at a repetition rate of about $10 \mathrm{~Hz}$ and a wall-plug efficiency of more than $10 \%{ }^{[18,19]}$. A $100 \mathrm{~J}$-class laser is now under development at the Central Laser Facility (CLF) in collaboration with HiLASE.

The system incorporates a low-energy, fiber-based front end oscillator $(\sim \mathrm{nJ})$, followed by a regenerative amplifier that increases the output energy to the $\mathrm{mJ}$ level and a thindisk $\mathrm{Yb}^{3+}$ :YAG multi-pass booster amplifier to raise the output to $100 \mathrm{~mJ}$. Two diode-pumped, helium-gas-cooled large-aperture power amplifiers then increase the output energy to between 7 and $10 \mathrm{~J}$ (Main Pre-amplifier) and finally to $100 \mathrm{~J}$ (Power Amplifier). The schematic of the system is shown in Figure 5.

\subsection{Front end}

The front end starts with a temperature-stabilized tunable $\mathrm{CW}$ fiber oscillator. The wavelength of the oscillator is matched to the peak of the gain curve of the cryogenically cooled amplifiers. The CW beam is then temporally shaped

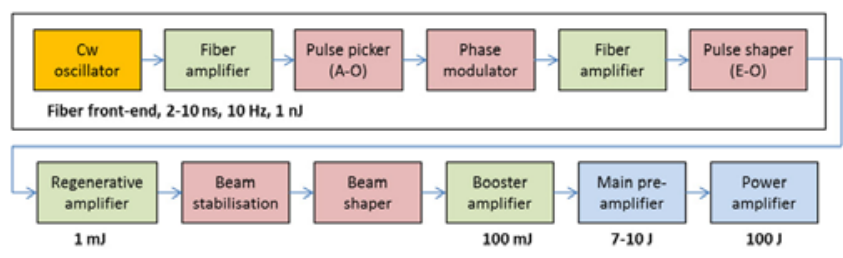

Figure 5. Schematic of the $100 \mathrm{~J}$ multi-slab laser system. The numbers represents the energy after the respective element.

in an acousto-optic (A-O) modulator to limit the repetition rate to $10 \mathrm{kHz}$ and subsequently shaped by an electro-optic (E-O) modulator to produce 2-10 ns pulses with a semitriangular shape. The temporal resolution of the shaper is below 200 ps. The pulses are further phase modulated by 2 and $4 \mathrm{GHz}$ modulators to increase the bandwidth of the pulses and prevent stimulated Brillouin scattering (SBS) and stimulated Raman scattering (SRS) in the amplifier chain. Then the repetition of the pulses is decreased by a pulse picker and pulses are amplified in a thin regenerative disk amplifier to $\sim 1 \mathrm{~mJ}$. The Gaussian beam coming from the regenerative amplifier is then spatially shaped to a square cross-section super-Gaussian profile in a beam shaper. Then it is further amplified to $\sim 100 \mathrm{~mJ}$ in a multi-pass booster amplifier. The booster amplifier preserves the square superGaussian beam profile that is injected into the $10 \mathrm{~J}$ main preamplifier.

\section{2. $10 \mathrm{~J}$ main pre-amplifer}

The $10 \mathrm{~J}$ main pre-amplifier is based on a multi-slab design. It consists of four circular $\mathrm{Yb}^{3+}$ :YAG slabs with two doping levels of $\mathrm{Yb}^{3+}(1.1,2.0$ at.\%). The different doping levels are needed to uniformly divide the heat load among the slabs. The volume of each circular slab is diameter in $45 \mathrm{~mm}$ with a thickness of $5 \mathrm{~mm}$ and the pumped area is square $23 \mathrm{~mm} \times 23 \mathrm{~mm}$. The pump beam is homogenized light from diode stacks operating at $939 \mathrm{~nm}$ and producing $700 \mu \mathrm{s}$ long laser pulses at a repetition rate of $10 \mathrm{~Hz}$. The $\mathrm{Yb}^{3+}: \mathrm{YAG}$ is clad with a $5 \mathrm{~mm} \mathrm{Cr}^{4+}$ :YAG absorber (absorption coefficient $6 \mathrm{~cm}^{-1}$ ) that prevents amplified spontaneous emission (ASE) and parasitic oscillations. The amplifier is cooled by forced helium gas flow and operates between 150 and $170 \mathrm{~K}$. 


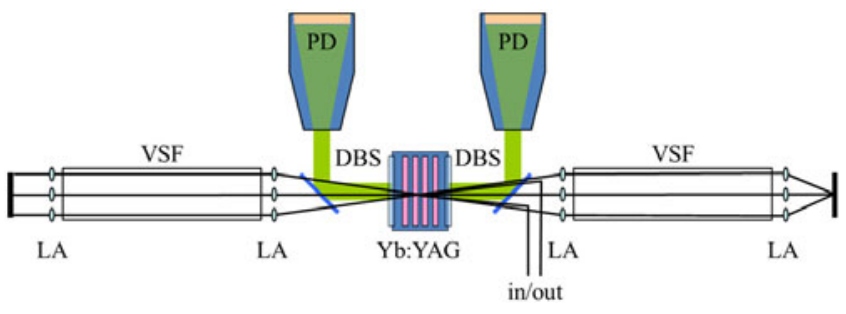

Figure 6. Schematic of the $10 \mathrm{~J}$ cryogenic multi-slab amplifier. It consists of $\mathrm{Yb}^{3+}$ :YAG ceramic slabs in the laser head (Yb:YAG), dichroic beam splitters (DBSs), lens arrays (LAs), vacuum spatial filters (VSFs), and homogenized pump diode laser modules (PDs).

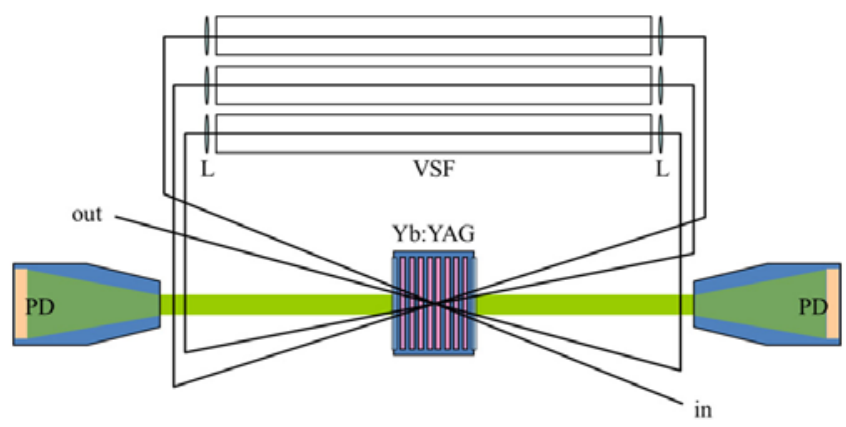

Figure 7. Schematic of the $100 \mathrm{~J}$ cryogenic multi-slab amplifier. It consists of $\mathrm{Yb}: \mathrm{YAG}$, lenses (L), VSF, and PD.

The extraction scheme of the multi-pass amplifier is shown in Figure 6. The beam is injected into the amplifier through a dichroic mirror and then image-relayed by a spatial filter $(f=1 \mathrm{~m})$ to a back reflector and back to the amplifier head. There is one spatial filter on each side of the amplifier head. Each pass is propagated by a set of separate mirrors. A deformable mirror is placed in the amplifier after the third pass. After seven or eight passes, the beam is ejected from the amplifier with a pulse energy of $7 \mathrm{~J}$ in the beam and a size of $20 \mathrm{~mm} \times 20 \mathrm{~mm}$.

\subsection{J power amplifier}

The $100 \mathrm{~J}$ power amplifier is also based on the multi-slab design. It consists of six square $\mathrm{Yb}^{3+}:$ YAG slabs with three doping levels of $\mathrm{Yb}^{3+}(0.4,0.6,1.0$ at.\%). The volume of each slab is $100 \mathrm{~mm} \times 100 \mathrm{~mm} \times 8.5 \mathrm{~mm}$ and the square pumped area is around $75 \mathrm{~mm} \times 75 \mathrm{~mm}$. The parameters of the pump light are similar to the $10 \mathrm{~J}$ amplifier. The $\mathrm{Yb}^{3+}$ :YAG is clad with a $10 \mathrm{~mm}$ wide $\mathrm{Cr}^{4+}: \mathrm{YAG}$ absorber (absorption coefficient $3 \mathrm{~cm}^{-1}$ ) that prevents ASE and parasitic oscillations.

The extraction scheme of the multi-pass amplifier is shown in Figure 7. The beam is injected into the amplifier at a range of angles around $5^{\circ}$, so any overlap with the pump beam outside the amplifier head is avoided and no dichroic mirrors are used. Each additional pass is image-relayed by a dedicated spatial filter $(f=3 \mathrm{~m})$ back to the amplifier head.
Table 2. Status of kW-class Multi-slab Beamline.

\begin{tabular}{ll}
\hline Laser system & Beamline A \\
\hline Completed & $10 \mathrm{~J}$ main pre-amplifier \\
Under development & $100 \mathrm{~J}$ power amplifier \\
Achieved energy & $10 \mathrm{~J}$ \\
Next milestone energy & $50 \mathrm{~J}$ \\
Final energy & $100 \mathrm{~J}$ \\
Operational & Q3 2015 \\
\hline
\end{tabular}

After the first pass, a deformable mirror is implemented to prevent degradation of the wavefront on subsequent passes. After four passes, the beam is ejected from the amplifier with a pulse energy of up to $120 \mathrm{~J}$ and a beam size around $75 \mathrm{~mm} \times 75 \mathrm{~mm}$. The average fluence in the amplified beam is $2 \mathrm{~J} \mathrm{~cm}^{-2}$. The amplifier is cooled by forced helium gas flow and operates between 150 and $170 \mathrm{~K}$.

The current status of the multi-slab laser system is indicated in Table 2.

\subsection{Numerical modeling}

The HiLASE team has undertaken extensive energetics, thermal and fluid-mechanical modeling in order to optimize various amplifier parameters.

For energetics modeling, we have developed a MATLAB code $^{[20]}$ for the evaluation of stored energy in the laser amplifier that includes ASE. This model calculates the pump energy absorbed by the gain medium, excited ion density, ASE and heat sources in the $\mathrm{Yb}^{3+}$ :YAG laser slabs by solving the rate equations in discrete time steps. During each step, the absorption of the pump radiation and spontaneous emission are calculated independently. In the absorption phase, the energy from a polychromatic pump source is absorbed in the medium in accordance with a probability proportional to the number of unexcited active ions and the absorption cross-section dependent on the pump wavelength. In the ASE phase, spontaneously emitted photons with random polarization are generated by the Monte Carlo method using the excited ion density inside the slab as the probability distribution. Their wavelength distribution is based on a probability density function derived from the emission crosssection. Rays containing thousands of photons are traced through the medium and amplified proportionally to the population inversion and the path length in each cell. All slabs of the amplifier are included in the model so the propagation of the rays among the slabs can be modeled. All surfaces can be treated as Fresnel reflecting, AR coated, or absorbing. Side faces can also include scattering. The slab model includes the active $\mathrm{Yb}^{3+}$ :YAG core and the absorbing $\mathrm{Cr}^{4+}$ :YAG cladding. A schematic of the slab and the calculated heat deposition are shown in Figure 8. Results were obtained for a slab size $100 \mathrm{~mm} \times 100 \mathrm{~mm}$, pump size $75 \mathrm{~mm} \times 75 \mathrm{~mm}, \mathrm{Cr}^{4+}$ :YAG cladding $10 \mathrm{~mm}$, 


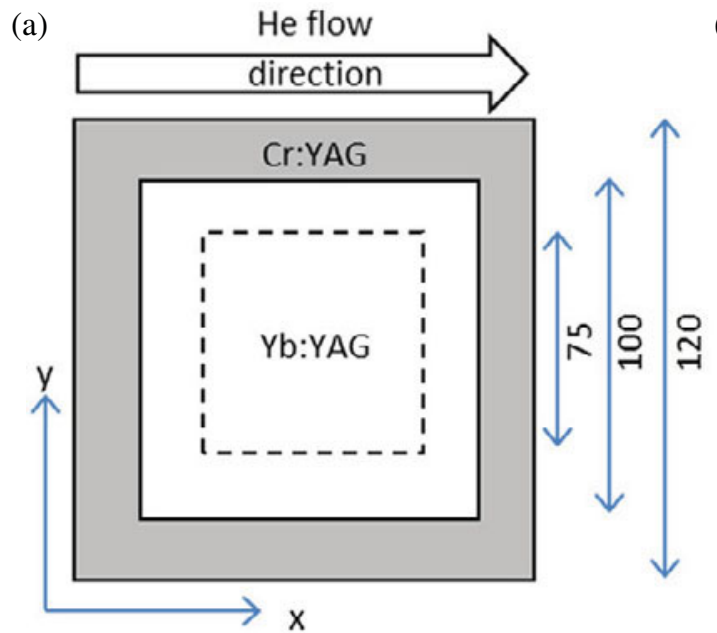

(b)

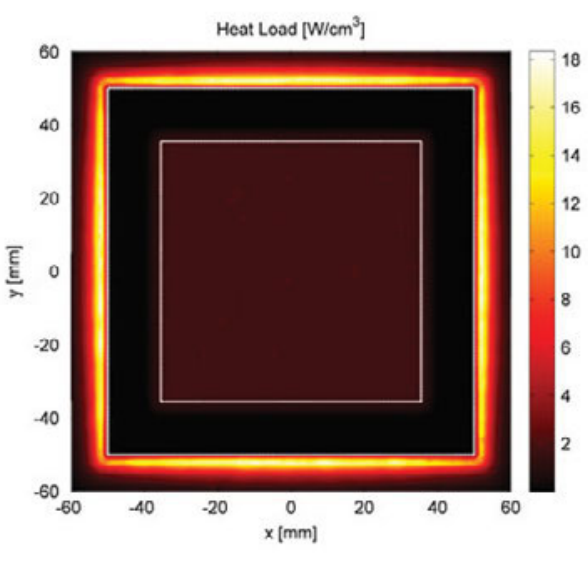

Figure 8. (a) Schematic of the laser slab with dimensions in mm, dashed line shows the spot of the pump beam in the $\mathrm{Yb}^{3+}: \mathrm{YAG}$ part of the slab that is clad by $\mathrm{Cr}^{4+}$ :YAG. (b) Transverse heat load of the slab used for the calculations (assumed constant in the longitudinal direction).

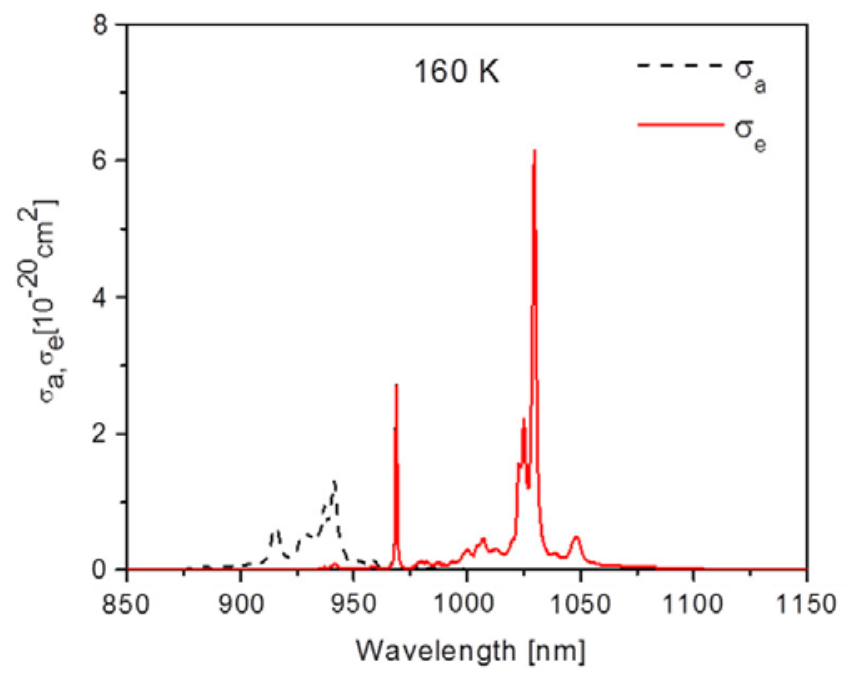

Figure 9. Measured absorption and emission cross-sections of the $\mathrm{Yb}^{3+}$ :YAG at a temperature of $160 \mathrm{~K}$.

absorption coefficient $3 \mathrm{~cm}^{-1}$, pump duration $1 \mathrm{~ms}$, intensity $5 \mathrm{~kW} \mathrm{~cm}^{-2}$, and temperature $160 \mathrm{~K}$.

The wavelength-resolved absorption and emission crosssections and lifetime on the upper laser level for a given temperature were obtained experimentally ${ }^{[21]}$. The crosssection and lifetime measurement station supports spectral resolution down to $15 \mathrm{pm}$ and temporal resolution down to 100 ps. An example of the measurement of the absorption and emission cross-section of $\mathrm{Yb}^{3+}: \mathrm{YAG}$ at a temperature of $160 \mathrm{~K}$ is shown in Figure 9.

A three-dimensional finite-element method (FEM) using Comsol Multiphysics software was chosen to model the thermal and stress effects in the amplifiers. The sources of heat were calculated in the ASE code. The lateral surfaces of the slabs are assumed to be cooled by flowing helium gas at $160 \mathrm{~K}$. The spatially resolved heat transfer coefficient was derived from a two-dimensional model of a turbulent flow of helium gas at $160 \mathrm{~K}$ using the standard $k \varepsilon$ model $^{[22]}$ together with Kays-Crawford heat exchange in the turbulent boundary layer ${ }^{[23,24]}$. The slab was assumed to have no thermal contact with its $2 \mathrm{~cm}$ thick Invar holder; hence all the heat is removed by convection through the faces. From the temperature and stress maps of the slab (Figure 10), optical path difference (OPD) and birefringence depolarization losses (Figure 11) were calculated for a single slab according to a prior approach ${ }^{[25]}$. The gradual decrease of heat exchange efficiency in the direction of gas flow, caused by coolant heating, is the reason for the loss of leftright symmetry of the temperature, stress, depolarization, and OPD maps.

A beam propagation model of the $100 \mathrm{~J}$ power amplifier was created in MIRÓ using a Fresnel diffraction integral for propagation and the Frantz-Nodvik equation for amplification. The model was used to estimate beam aberrations, taking into consideration only the thermal OPD. The results of the beam intensity and OPD are shown in Figure 12. The resulting wavefront was corrected numerically. The reliability of the wavefront correction code was verified experimentally in a slab simulator ${ }^{[26]}$.

The numerical model for wavefront correction calculates influence functions from a plate equation describing the bending of the thin facesheet for each individual actuator of the deformable mirror. The deformable mirror consists of a continuous gold facesheet (size of $100 \mathrm{~mm} \times 100 \mathrm{~mm}$, thickness of $1 \mathrm{~mm}$ ) on which lateral forces are applied by piezoelectric stack actuators. The actuators form an equidistantly spaced rectangular array of $6 \times 6$ actuators and are capable of push/pull operation. The deformation of the mirror is computed as a superposition of the influence functions and the algorithm minimizes the rms OPD value. 
(a)

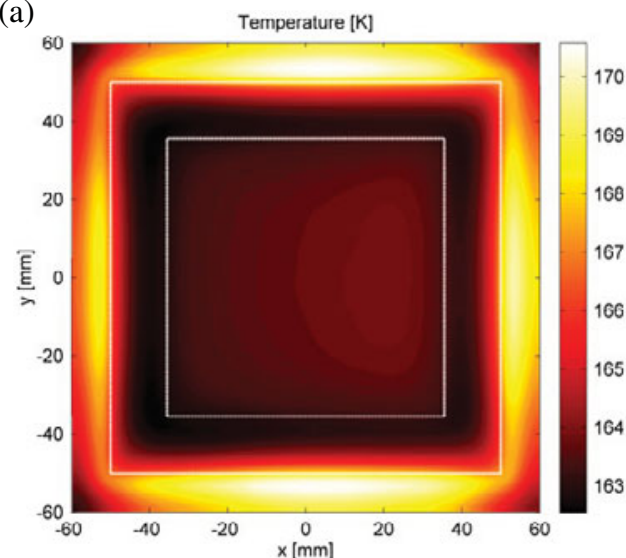

(b)

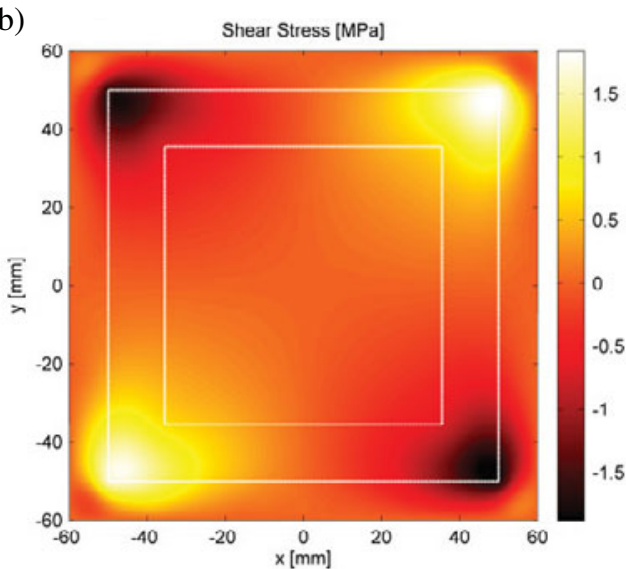

Figure 10. (a) Transverse distribution of temperature and (b) transverse distribution of the $x y$ stress component in a longitudinal cut in the center of the laser slab.

(a)

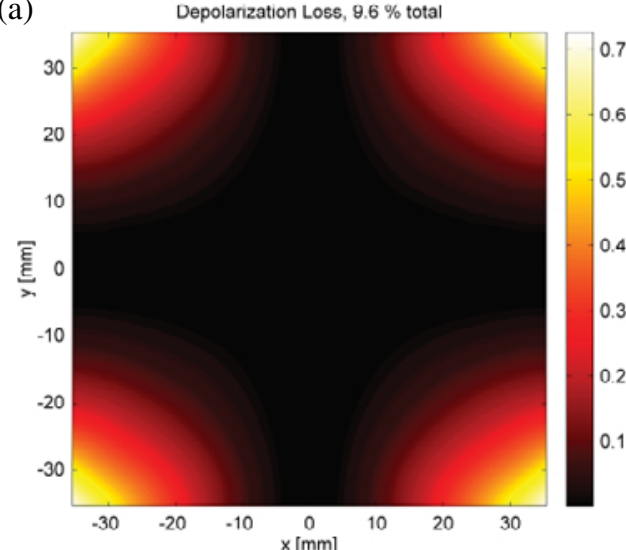

(b)

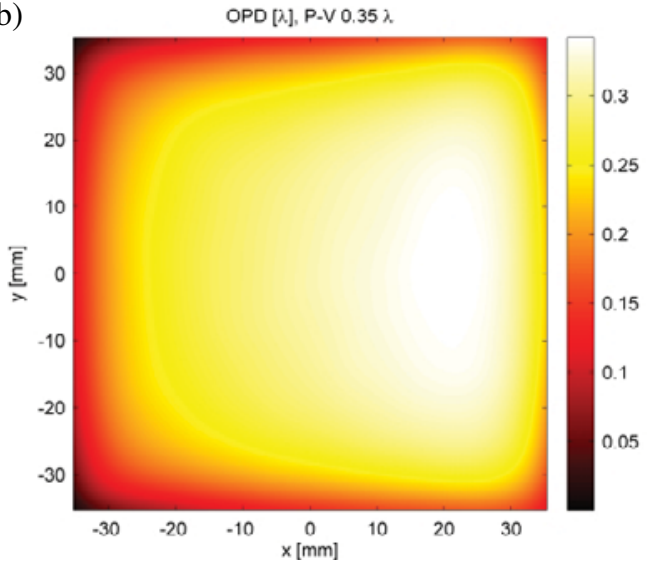

Figure 11. (a) Depolarization of the beam at the output of the amplifier (after four passes through six laser slabs) caused by stress-induced birefringence. (b) Stress- and temperature-induced OPD after a single pass through the laser head (after one pass through six laser slabs).
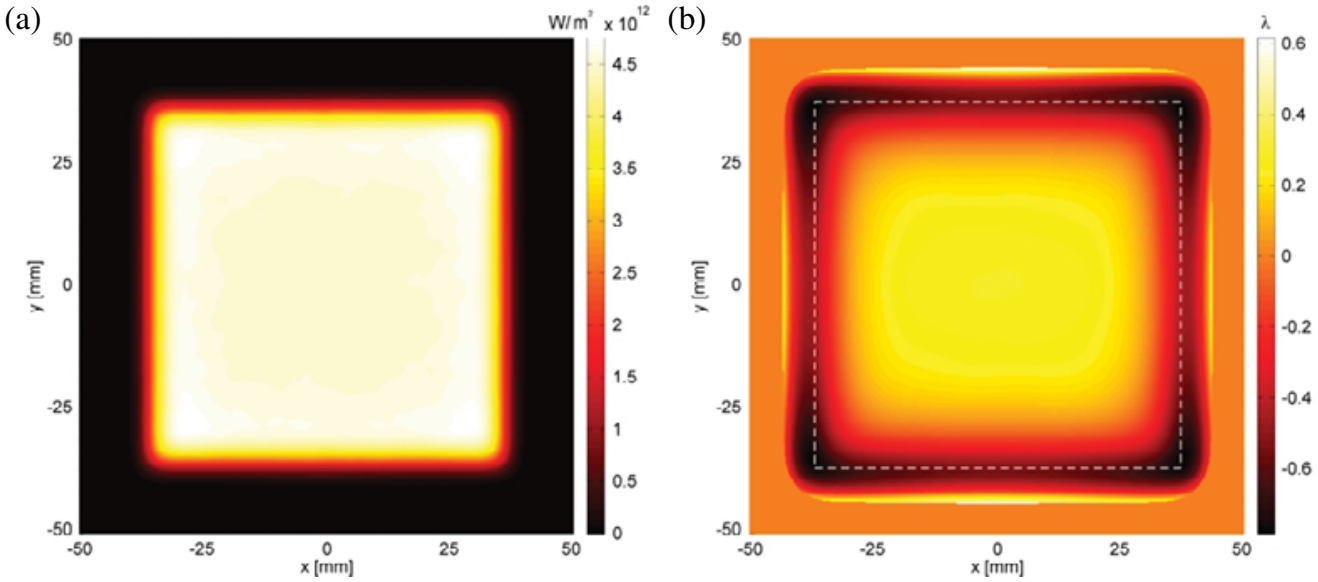

Figure 12. (a) Beam profile and (b) OPD of the beam at the output of the $100 \mathrm{~J}$ multi-slab system calculated in MIRÓ. Dashed square indicates the position of the laser beam.

The OPD after subtraction of defocus and tilt and the OPD corrected by the deformable mirror are shown in Figure 13.
The rms value of the OPD was reduced from $111 \mathrm{~nm}$ down to $31 \mathrm{~nm}$. 
(a)

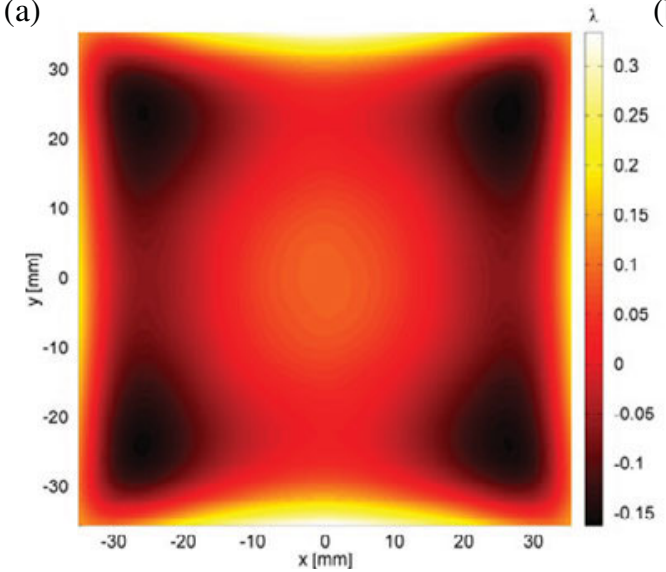

(b)

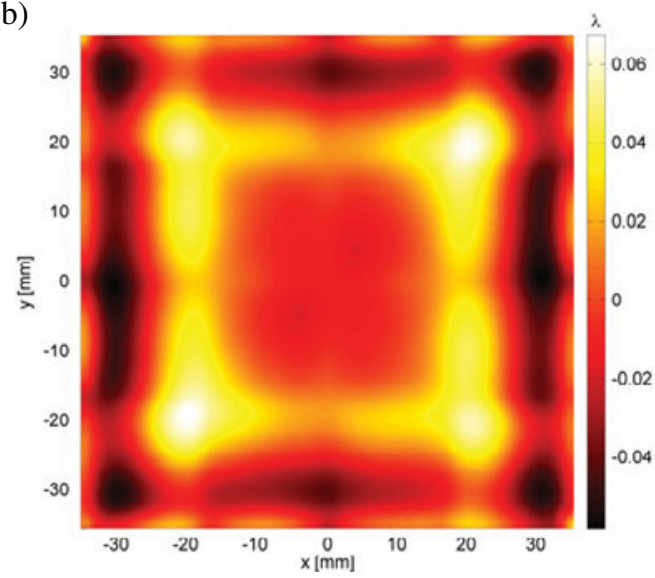

Figure 13. (a) Output OPD calculated in MIRÓ and shown in Figure 12(b) after subtraction of tilt and defocus. (b) Residual OPD after correction by the deformable mirror with 36 actuators.

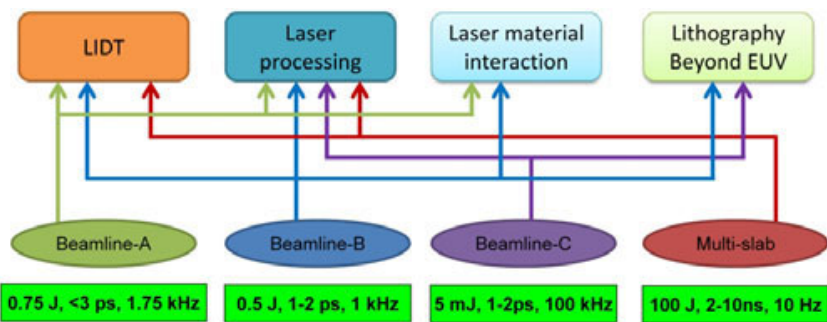

Figure 14. Schematic of the HiLASE application program.

\section{Applications}

One of the long-term objectives of HiLASE is the identification of new and promising industrial applications and technologies using the DPSSL systems that were described above. Once commissioned in the HiLASE center these advanced DPSSL systems will enable, for example, research relevant to the testing of new dielectric optical components with high damage thresholds, prototyping new pump lasers for OPCPA (Optical Parametric Chirped Pulse Amplification) systems, driving high yield secondary photon and particle sources, and industrial applications related to efficient processing of materials (ablative removal of thin layers, cutting of optically transparent materials, laser peening, surface structuring and modifications, etc.). An overview of the HiLASE laser application program is shown in Figure 14.

\subsection{Laser-induced damage threshold testing}

First, the laser-induced damage threshold (LIDT) automated experimental station would be introduced. The station design allows one to measure the LIDT under a wide range of laser parameters: from the irradiation of small spots with $1-2 \mathrm{ps}$ laser pulses at various wavelengths and a $1 \mathrm{kHz}$ repetition rate to the irradiation of large spots with 2-10 ns laser pulses at $1030 \mathrm{~nm}$ with a $10 \mathrm{~Hz}$ repetition rate. The main advantage

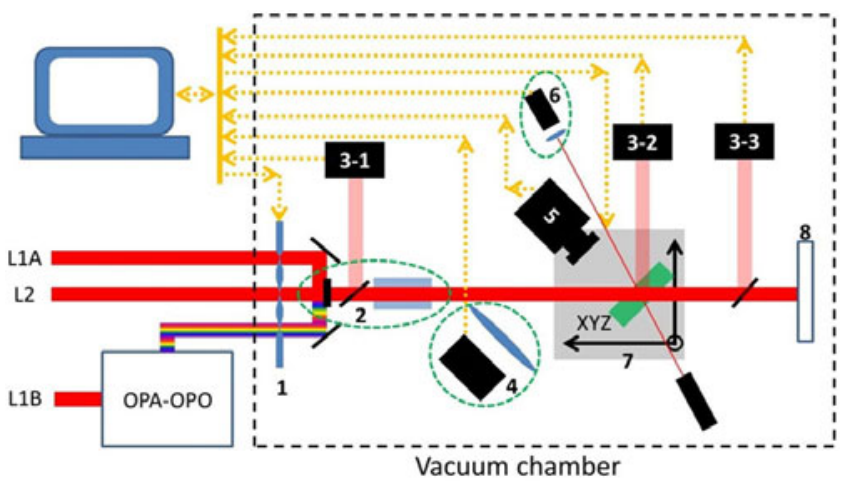

Figure 15. Schematic of the LIDT measurement station: (1) high-speed shutter, (2) beam positioning and focus, (3) beam diagnostics, (4) scattered light damage detection and fluorescence collector, (5) slow-motion camera, (6) interference damage detection; (7) XYZ tower, (8) beam dump. It uses laser pulses from Beamline A (L1A), Beamline B (L1B), and the Multi-slab (L2) laser system.

of this station is real-time monitoring of laser damage with an acquisition frequency of up to $1 \mathrm{kHz}$. This station will allow the determination of the damage occurrence, as well as following the damage growth and damage threshold variation under repetitive irradiation. A schematic of the station is shown in Figure 15. The particular design of the LIDT station permits investigations of samples of up to $1 \mathrm{~kg}$ with surface sizes of $100 \mathrm{~mm} \times 100 \mathrm{~mm}$.

\subsection{Mid-IR optical parametric generator}

For the investigation of laser-material interactions and processing, as well as the thin-disk and multi-slab systems, a mid-IR pulse source with a high repetition rate and an average power of $10 \mathrm{~W}^{[27]}$ is being developed. The proposed scheme of the wavelength conversion setup (Figure 16) uses the picosecond output from the thin-disk regenerative amplifier as the pump beam. The seed beam for the wave- 


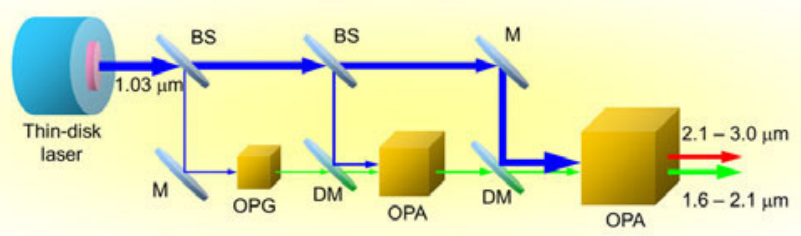

Figure 16. Schematic of the mid-IR parametric generator and amplifier. It consists of the thin-disk laser system, beam splitters (BS), mirrors (M), dichroic mirrors (DM), an OPG, and OPA.

length conversion setup comes from the optical parametric generator (OPG) and the signal is tunable between 1.6 and $2.1 \mu \mathrm{m}$. Only the signal beam is amplified by the optical parametric amplifier (OPA) chain. Idler beams having wavelengths from 2 to $3 \mu \mathrm{m}$ can be extracted from the last parametric amplifier.

\subsection{EUV light generation}

For EUV generation, powerful $\mathrm{CO}_{2}$ lasers are used to evaporate tin $(\mathrm{Sn})$ droplets to generate a plasma that emits light at $13.5 \mathrm{~nm}$. The $\mathrm{CO}_{2}$ laser provides a much higher average power and higher conversion efficiency to UV light, but the laser footprint and plasma size are large ${ }^{[28]}$, so it is suited for high-volume manufacturing. There is also a need for a smallscale EUV source with very high brightness for method and component testing. This compact source requires a stable beam with a higher beam quality than the beam quality provided by $\mathrm{CO}_{2}$ lasers but at the same time the average power output required is only in the region of $1 \mathrm{~kW}$. For this reason, we are constructing an EUV generation station using a thindisk amplifier with a high average power. This source will be employed to study processes during EUV light generation.

\section{Acknowledgements}

This work benefited from the support of the Czech Republic's Ministry of Education, Youth and Sports to the HiLASE (CZ.1.05/2.1.00/01.0027), DPSSLasers (CZ.1.07/2.3.00/ 20.0143), and Postdok (CZ.1.07/2.3.00/30.0057) projects, co-financed by the European Regional Development Fund. This research has been partially supported by grant RVO 68407700 .

\section{References}

1. E. Shcherbakov, V. Fomin, A. Abramov, A. Ferin, D. Mochalov, and V. P. Gapontsev, In Advanced Solid-State Lasers Congress (Optical Society of America, Washington, DC, 2013) ATh4A.2.

2. Joint High Power Solid-State Laser, http://www.northropgru mman.com/Capabilities/SolidStateHighEnergyLaserSystems/
Pages/JointHighPowerSolidStateLaser.aspx

(January 29, 2014).

3. T. Metzger, A. Schwarz, C. Teisset, D. Sutter, A. Killi, R. Kienberger, and F. Krausz, Opt. Lett. 34, 2123 (2009).

4. C. Y. Teisset, M. Schultze, R. Bessing, M. Häfner, S. Prinz, D. Sutter, and T. Metzger, In Advanced Solid-State Lasers Congress (Optical Society of America, Washington, DC, 2013) JTh5A.1.

5. R. Jung, J. Tümmler, Th. Nubbemeyer, I. Will, W. Sandner, G. Erbert, and W. Pittroff, Disk lasers for powerful picosecond pulses with $100 \mathrm{~Hz}$ repetition rate. In 2nd Disk Laser Workshop (Dausinger and Giessen, Stuttgart) (2012).

6. J.-P. Negel, A. Voss, M. A. Ahmed, D. Bauer, D. Sutter, A. Killi, and T. Graf, Opt. Lett. 38, 5442 (2013).

7. C. J. Saraceno, F. Emaury, C. Schrieber, M. Hoffmann, M. Golling, T. Sudmeyer, and U. Keller, Opt. Lett. 39, 9 (2014).

8. M. Hornung, S. Keppler, R. Bodefeld, A. Kessler, H. Liebetrau, J. Koerner, M. Hellwing, F. Schorcht, O. Jackel, A. Savert, J. Polz, A. K. Arunachalam, J. Hein, and M. C. Kaluza, Opt. Lett. 38, 718 (2013).

9. M. Divoky, S. Tokita, H. Furuse, K. Matsumoto, Y. Nakamura, and J. Kawanaka, In Advanced Solid-State Lasers Congress (Optical Society of America, Washington, DC, 2013) AF2A.5.

10. T. Goncalves-Novo, D. Albach, B. Vincent, M. Arzakantsyan, and J. C. Chanteloup, Opt. Express 21, 855 (2013).

11. K. Ertel, S. Banerjee, P. Mason, P. Phillips, R. Greenhalgh, C. Hernandez-Gomez, and J. Collier, Proc. SPIE 8780, 87801W (2013).

12. A. Bayramian, S. Aceves, T. Anklam, K. Baker, E. Bliss, C. Boley, A. Bullington, J. Caird, D. Chen, R. Deri, M. Dunne, A. Erlandson, D. Flowers, M. Henesian, J. Latkowski, K. Manes, W. Molander, E. Moses, T. Piggott, S. Powers, S. Rana, S. Rodriguez, R. Sawicki, K. Schaffers, L. Seppala, M. Spaeth, S. Sutton, and S. Telford, Fusion Sci. Technol. 60, 28 (2011).

13. A. Giesen, H. Hugel, A. Voss, K. Wittig, U. Brauch, and H. Opower, Appl. Phys. B 58, 365 (1994).

14. C. Kränkel, R. Peters, K. Petermann, P. Loiseau, G. Aka, and G. Huber, J. Opt. Soc. Am. B 26, 1310 (2009).

15. M. Chyla, T. Miura, M. Smrz, P. Severova, O. Novak, S.S. Nagisetty, A. Endo, and T. Mocek, Proc. SPIE 8780, 87800A (2013).

16. M. Smrz, T. Miura, M. Chyla, A. Endo, and T. Mocek, In IEEE Photonics Conference (IEEE, Washington, 2013) MD1.4.

17. A. Bayramian, J. Armstrong, G. Beer, R. Campbell, B. Chai, R. Cross, A. Erlandson, Y. Fei, B. Freitas, R. Kent, J. Menapace, W. Molander, K. Schaffers, C. Siders, S. Sutton, J. Tassano, S. Telford, C. Ebbers, J. Caird, and C. Barty, J. Opt. Soc. Am. B 25, B57 (2008).

18. A. C. Erlandson, S. M. Aceves, A. J. Bayramian, A. L. Bullington, R. J. Beach, C. D. Boley, J. A. Caird, R. J. Deri, A. M. Dunne, D. L. Flowers, M. A. Henesian, K. R. Manes, E. I. Moses, S. I. Rana, K. I. Schaffers, M. L. Spaeth, C. J. Stolz, and S. J. Telford, Opt. Mater. Express 1, 1341 (2011).

19. P. D. Mason, K. Ertel, S. Banerjee, P. Phillips, C. HernandezGomez, and J. Collier, Proc. SPIE 8780, 87801X (2013).

20. M. Sawicka, M. Divoky, J. Novak, A. Lucianetti, B. Rus, and T. Mocek, J. Opt. Soc. Am. B 29, 1270 (2012).

21. V. Jambunathan, J. Koerner, P. Sikocinski, M. Divoky, M. Sawicka, A. Lucianetti, J. Hein, and T. Mocek, Proc. SPIE 8780, 87800G (2013). 
22. B. E. Launder and D. B. Spalding, Comput. Meth. Appl. Mech. Eng. 3, 269 (1974).

23. W. M. Kays, Convective Heat and Mass Transfer 3rd edition (McGraw-Hill Inc., 1993).

24. H. Schlichting and K. Gersten, Boundary Layer Theory 8th edition (Springer Verlag, Berlin, 2000).

25. O. Slezak, A. Lucianetti, M. Divoky, M. Sawicka, and T. Mocek, IEEE J. Quantum Electron. 49, 960 (2013).
26. J. Pilar, M. Divoky, P. Sikocinski, O. Slezak, A. Lucianetti, V. Kmetik, S. Bonora, and T. Mocek, Proc. SPIE 8780, 878011 (2013).

27. O. Novak, T. Miura, P. Severova, M. Smrž, A. Endo, and T. Mocek, In Advanced Solid-State Lasers Congress (Optical Society of America, Washington, DC, 2013) JTh2A.29.

28. A. Endo, In Source: Lithography M. Wang, (eds.) (INTECH, Croatia, 2010) chap. 9. 